\section{Termine}

\section{Köln, 13. Juni 2001}

Allergie-Symposium im Rahmen der ÄDA-Regionalgruppe Köln

\section{Themen:}

- Neuere Entwicklungen der allergologischen In-vitro-Diagnostik (Prof. Dr. H.F. Merk, Aachen)

- Neue Aspekte von Autoimmunerkrankungen (PD Dr. N. Hunzelmann, Köln)

\section{Veranstaltungsort:}

Hörsaal 2, LFI-Gebäude des Universitätsklinikums, Joseph-Stelzmann-Str. 9

Beginn: $18.00 \mathrm{Uhr}$

Organisation:

Prof. Dr. K. Scharffetter-Kochanek Klinik und Poliklinik für Dermatologie und Venerologie der Universität zu Köln, Joseph-Stelzmann-Str. 9 D-50924 Köln

Tel.: $0221 / 478-5086$

Fax: $0221 / 478$ - 4549

\section{Rotenburg (Wümme), 25. August 2001}

4. Rotenburger AllergieSymposium

Veranstalter: Lungenklinik Unterstedt und ÄDA (Regionalgruppe Niedersachsen-Nord)

\section{Themen:}

_ In-vitro-Teste: Was ist sinnvoll? Was ist überflüssig?

_ Häusliche Allergene: Was gibt es außer Hausstaubmilben?

_ Primär- und Sekundärprävention in der Allergologie: Stand der Dinge und der Möglichkeiten

_ Vocal cord dysfunction-Syndrom

— Streitgespräch: Prick- vs. Intrakutan-Testung - welche Technik bei welcher Fragestellung?

Organisation: Prof. Dr. T. Schaberg Lungenklinik Unterstedt Verdenerstr. 200 D-27342 Rotenburg (Wümme) Tel.: $04261 / 776235$ Fax: $04261 / 776208$

\title{
Der Mai brachte eine neue BG-GOÄ
}

\author{
Wer mit einer Vereinfachung gerechnet hat, wird enttäuscht. Wir \\ müssen unverändert mit drei Gebührenordnungen leben: EBM, \\ GOÄ 96 und der neuen BG (UV)-GOÄ.
}

$\mathrm{D}$ ie neue Gebührenordnung BGGOÄ ist ein Cocktail aus GOÄ 96, GOÄ 88 (z.B. hausärztliche Ziffern) und neuen Ziffern. Unglücklicherweise teilen sich nun „Untersuchung" und „Beratung“ die Ziffer 1 dafür ist die Untersuchung jetzt "Symptomen-zentriert". Der untersuchende und beratende Arzt bekommt für beide Komplexe satte 12,15 DM anstelle von 18,24 DM (GOÄ 96 ). Insgesamt kann man dieses Werk nicht als den großen Wurf bezeichnen - eher als Rückschritt in das Jahr 1988.

Etwas Positives verbleibt für die gutachterlich tätigen Kollegen. Die Gutachter-Gebühren mit den verschiedenen Schwierigkeitsgraden sind jetzt höher, ebenso die Schreibgebühren. Gutachter können ärztliche Leistungen in Zusammenhang mit Begutachtungen mit Spalte 3 (besondere Heilbehandlung) abrechnen. Die Ziffern der einzelnen Leistungen entsprechen weitgehend denen der GOÄ 96. Für reine Begutachtungen können wir darin eine Vereinfachung sehen, da für Sozialgerichte, Privatpatienten und BG-Versicherte die gleichen Ziffern für diesen Bereich gelten.

Es gilt also ein neues Tabellenwerk zu studieren, die Computer entsprechend umzustellen und mit neuen Programmen zu füttern und dies wie immer auf eigene Kosten!

Wenn alles dann gut eingeübt ist, können wir die nächste Gebührenordnung freudig erwarten.

Dr.W. Kersten, Moers

\section{KBV fordert morbiditätsbezogenes Richtgrößenkonzept}

K

BV-Vorsitzender Dr. Manfred Richter-Reichhelm hat die Krankenkassen aufgefordert, noch im laufenden Jahr mit der KBV konkrete Vereinbarungen zu treffen, damit das Arzneimittelbudget-Ablösungsgesetz (ABAG) 2002 umgesetzt werden kann.

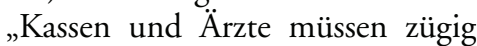
ein morbiditätsbezogenes Richtgrößenkonzept erarbeiten“, erklärte der KBVChef. „Die KBV ist bereit, ihren Anteil an der gemeinsamen Verantwortung für die Arzneimittelversorgung zu übernehmen. Bundesgesundheitsministerin Schmidt und wir sind uns einig “, unterstrich Richter-Reichhelm nach einem Gipfelgespräch im Ministerium.

Trotz der mit der KBV ausgehandelten Bundesempfehlungen gingen die GKV-Spitzenverbände auf Distanz zur Ministerin. Sie machen die Koalition für die „Zuspitzung der finanziellen
Situation der GKV“ verantwortlich. Trotz erheblicher Anstrengungen seitens der Kassen sei eine Konsolidierung der GKV-Finanzen nicht erreicht worden. Grund dafür seien kontinuierlich neu auf die Kassen verlagerte Belastungen durch den Gesetzgeber und die Rechtsprechung, meinen die GKV-Verbände. Eine konkrete Forderung: „Erheblich entlastend würde die Reduzierung der Mehrwertsteuer auf Arzneimittel sowie die sachgerechte Steuerfinanzierung versicherungsfremder Leistungen wirken."

Die Aufhebung der Arzneibudgets und des Kollektivregresses halten die Krankenkassen dagegen unverändert für „belastend“. Die Ausgabensteuerung über Budgets sei notwendig, könne aber „durch den Abschluss von Zielvereinbarungen flankiert“ werden.

red 